\title{
Optimal Teleportation with a Mixed State of Two Qubits
}

\author{
Frank Verstraete ${ }^{1,2}$ and Henri Verschelde ${ }^{2}$ \\ ${ }^{1}$ Department of Electrical Engineering (ESAT-SISTA), KULeuven, Leuven, Belgium \\ ${ }^{2}$ Department of Mathematical Physics and Astronomy, Ghent University, B-9000 Gent, Belgium
}

(Received 27 August 2002; published 5 March 2003)

\begin{abstract}
We consider a single copy of a mixed state of two qubits and derive the optimal trace-preserving local operations assisted by classical communication such as to maximize the fidelity of teleportation that can be achieved with this state. These optimal local operations turn out to be implementable by one-way communication and always yield a teleportation fidelity larger than $2 / 3$ if the original state is entangled. This maximal achievable fidelity is an entanglement measure and turns out quantifying the minimal amount of mixing required to destroy the entanglement in a quantum state.
\end{abstract}

DOI: 10.1103/PhysRevLett.90.097901

The basic resource in quantum information theory consists of maximally entangled qubits or Bell states. This stems from the fact that the additional resource of entanglement enables one to implement all possible global quantum operations locally by making use of the concept of quantum teleportation [1]. Perfect teleportation is possible only when maximally entangled states are available. In practical situations, however, we have to deal with mixed states due to the undesired coupling of the quantum states with the environment. In this Letter we address the following basic question: given a mixed state of two qubits, what is the maximal teleportation fidelity that can be obtained with this state allowing all possible tracepreserving local operations assisted by classical communication (LOCC) or all possible filtering operations (SLOCC)? We give a complete answer to those questions.

If only local unitary operations are allowed, then the Horodecki's [2] proved that the optimal teleportation fidelity $f$ is a linear function $f=(2 F+1) / 3$ of the maximal singlet fraction or fidelity $F$ [3], which is defined as the maximal overlap of a state with a maximally entangled (ME) state:

$$
F(\rho)=\max _{|\psi\rangle=\mathrm{ME}}\langle\psi|\rho| \psi\rangle .
$$

Therefore the problem of maximizing the teleportation fidelity is equivalent to maximizing the maximal singlet fraction of a mixed state of two qubits. This problem is also of great interest in the context of distillation protocols [3-6], as the distillation of barely entangled states occurs through the use of recurrence schemes which gradually enhance the fidelity under the condition that the initial fidelity $F$ exceeds $1 / 2$. The maximal fidelity for separable states is indeed given by $F=1 / 2$. Surprisingly, there exist entangled states whose fidelity is lower than this value [7], but the Horodecki's [8] proved that local filtering operations can always be chosen such that, with a finite probability, a state with fidelity exceeding $1 / 2$ is obtained if the original state was entangled. In this Letter we prove the stronger result that a mixed state of two qubits is entangled if and only if there exist trace-
PACS numbers: 03.67.Hk

preserving LOCC operations such that the fidelity $F^{*}$ of the LOCC-processed state exceeds $1 / 2$. This answers the following question raised by Badziag and Horodecki [9] in the case of two qubits: "Can any entangled state provide better than classical fidelity of teleportation?"

The present work also sheds new light on the open problem of characterizing the class of local operations that can physically be implemented on a system. Finding a parametrization of the class of LOCC operations turns out to be very difficult. The class of positive partial transpose (PPT) operations [10], however, related to the concept of partial transposition [11,12], is very easy to characterize but contains operations that cannot be implemented locally. In this letter, we consider the problem of maximizing the fidelity under the action of all PPT operations. Surprisingly, it turns out that the optimal PPT protocol is always physically implementable: this is supporting evidence for the fact that the class of PPT operations yields a good approximation of the class of LOCC operations.

In the first part, the optimal local filtering operations [13] such as to maximize the fidelity of the filtered state are derived. The optimal filter is the one that transforms the state into a unique Bell-diagonal form. This provides a quantitative way of characterizing the qualitative result of the work of the Horodecki's [8]. The drawback of filtering operations is the fact that these operations can be implemented only with a certain probability. It is therefore an interesting question whether trace-preserving local operations can also enhance the fidelity. In a surprising paper by Badziag et al. [9], it was shown that there exist mixed states with fidelity smaller than $1 / 2$, for which local trace-preserving protocols exist that transform this state into a state with fidelity larger than $1 / 2$ without the help of classical communication. Motivated by this example, we looked for the optimal LOCC protocols such as to transform an entangled state into one with fidelity as large as possible allowing classical communication. We prove that the optimal trace-preserving protocol for maximizing the fidelity of a given state always belongs to a very simple class of one-way communication (1-LOCC) 
operations, and we provide a constructive way of obtaining this optimal (state-dependent) LOCC operation. We conclude by giving a geometrical interpretation of the maximum achievable fidelity by LOCC, revealing an interesting connection with the robustness of entanglement [14].

Let us now state the first theorem of this Letter:

Theorem: The bipartite local filtering operations probabilistically bringing an entangled mixed state of two qubits to a state with the highest possible fidelity are given by the filtering operations bringing the state into its unique Bell-diagonal normal form [15], yielding a fidelity larger than $1 / 2$.

Proof: In [15] it was proven that the local filtering operations maximizing the concurrence [16] of a state are given by the local operations bringing the state into its unique Bell-diagonal normal form, and that a state is entangled if and only if its normal form is entangled. The fidelity of a state is bounded above by $F(\rho) \leq[1+$ $C(\rho)] / 2$ [7] with $C(\rho)$ the concurrence, and for Belldiagonal states the equality holds. It is moreover trivial to check that the fidelity of an entangled Bell-diagonal state exceeds $1 / 2$, which ends the proof.

Next we want to investigate if there always exist tracepreserving local operations such that the fidelity of the obtained state exceeds $1 / 2$ if the original state is entangled. The crucial point is to incorporate the previously described filtering operation as part of a trace-preserving LOCC operation. The idea is that it is always possible to make a trace-preserving LOCC operation out of a SLOCC filtering operation by making a pure separable state if the state did not pass the filter. Then with a certain probability a Bell-diagonal state $\rho_{f}$ arises with fidelity exceeding $1 / 2$, and with the complementary probability a pure separable state $|\chi\rangle$ can be created having fidelity equal to $1 / 2$ (note that $|\chi\rangle$ must be chosen such that $|\langle\chi \mid \psi\rangle|^{2}=$ $1 / 2$ with $|\psi\rangle$ the maximally entangled state obeying $\left.F\left(\rho_{f}\right)=\left\langle\psi\left|\rho_{f}\right| \psi\right\rangle\right)$. This proves that for each entangled mixed state of two qubits there exists a trace-preserving 1-LOCC protocol that transforms it into a state with fidelity larger than $1 / 2$.

Let us now try to optimize the trace-preserving operation used in the protocol just described such as to maximize the fidelity of a given state. Note that in general the optimal filter of theorem 1 will not be optimal in the trace-preserving setting as in that case the probability of obtaining the state was not taken into account. The setting is now as follows: we want to find the filter, such that the probability of success $p_{A B}$ of the filter multiplied by the fidelity $F$ of the state coming out of this filter, plus $\left(1-p_{A B}\right)$ times the fidelity of the pure separable state given by $1 / 2$, is maximal. For a given filter $-I \leq A, B \leq$ $I$, the cost function $K_{A B}$ is therefore given by

$$
K_{A B}=p_{A B} F\left(\rho_{f}\right)+\frac{1-p_{A B}}{2},
$$

where

$$
\begin{aligned}
p_{A B} & =\operatorname{Tr}\left((A \otimes B) \rho(A \otimes B)^{\dagger}\right), \\
\rho_{f} & =\frac{(A \otimes B) \rho(A \otimes B)^{\dagger}}{p_{A B}} .
\end{aligned}
$$

Now some tricks will be applied. Because of the presence of $A, B$, we can replace $F\left(\rho_{f}\right)$ by $\left\langle\psi\left|\rho_{f}\right| \psi\right\rangle$ with $|\psi\rangle=$ $(|00\rangle+|11\rangle) / \sqrt{2}$, and we use the fact that the trace of the product of two matrices is equal to the trace of the product of the partial transpose of two matrices. This leads to the following expression (see also the proof of theorem 1 in [7]):

$$
K_{A B}=\frac{1}{2}-\left\langle\psi\left|(C \otimes I) \rho^{\Gamma}\left(C^{\dagger} \otimes I\right)\right| \psi\right\rangle,
$$

where $C=B^{\dagger} \sigma_{y} A$ and $\rho^{\Gamma}$ is the shortcut notation for the partial transpose with respect to the system $B$. This cost function has to be maximized over all complex $2 \times 2$ matrices $-I \leq A, B \leq I$, and this leads to a lower bound on the maximum achievable fidelity by LOCC operations. Note that the considered operations can always be implemented using 1-LOCC, as one can always choose $B=I$ without loss of fidelity.

An upper bound can be obtained by using the techniques developed by Rains [10]. Indeed, if we enlarge the class of allowed operations from trace-preserving LOCC operations to trace-preserving PPT operations, a simple optimization problem arises. A quantum operation $\Lambda$ is PPT if and only if the dual state $\rho_{\Lambda}$ associated to this operation [2,10,17-19] is PPT. The dual state $\rho_{\Lambda}$ corresponding to a map $\Lambda$ on two qubits is defined in a $2 \otimes$ $2 \otimes 2 \otimes 2$ Hilbert space and the following relation holds:

$$
(\Lambda(\rho))_{A^{\prime} B^{\prime}}^{T}=4 \operatorname{Tr}_{A B}\left(\rho_{\Lambda}^{A A^{\prime} B B^{\prime}}\left(\rho_{A B} \otimes I_{A^{\prime} B^{\prime}}\right)\right) .
$$

An upper bound on $F^{*}$ can now be obtained by considering the following optimization problem: maximize

$$
4 \operatorname{Tr}\left(\rho_{\Lambda}(\rho \otimes|\psi\rangle\langle\psi|)\right)
$$

under the constraints

$$
\begin{aligned}
& \rho_{\Lambda} \geq 0, \quad \rho_{\Lambda}^{T_{B B^{\prime}}} \geq 0, \quad 4 \operatorname{Tr}_{A^{\prime} B^{\prime}}\left(\rho_{\Lambda}\right)=I_{A B} \\
& 4 \operatorname{Tr}_{A^{\prime} B^{\prime}}\left(\rho_{\Lambda}\right)=I_{A B}
\end{aligned}
$$

and with $|\psi\rangle$ a maximally entangled state. Here the notation $\rho^{T_{B B^{\prime}}}$ denotes the partial transpose with respect to the systems $B$ and $B^{\prime}$. This is a semidefinite program and can easily be solved numerically with guaranteed convergence [20]. Exploiting symmetries, however, it is possible to reduce the complexity drastically. Indeed, $|\psi\rangle$ remains invariant under a twirl operation and this twirl can be applied to $\rho_{\Lambda}$, leading to a state of the form

$$
\rho_{\Lambda}=\frac{1}{16}\left(I_{4} \otimes I_{4}+\left(4 X-I_{4}\right) \otimes\left(4|\psi\rangle\langle\psi|-I_{4}\right)\right) .
$$

Here $X$, a $4 \times 4$ matrix, is subject to convex constraints $I / 6 \leq X \leq I / 2$ and $0 \leq X^{\Gamma} \leq I / 3$. Doing the substitution $X \rightarrow\left(I-X^{\Gamma}\right) / 3$, this optimization problem reduces to the following semidefinite program (see also Rains [10]): maximize 


$$
1 / 2-\operatorname{Tr}\left(X \rho^{\Gamma}\right)
$$

under the constraints

$$
0 \leq X \leq I_{4}, \quad-\frac{I_{4}}{2} \leq X^{\Gamma} \leq \frac{I_{4}}{2} .
$$

Note that the constraint $-\frac{I_{4}}{2} \leq X^{\Gamma}$ will automatically be satisfied if the other three constraints are satisfied: this follows from the fact that $X^{\Gamma}$ has at most one negative eigenvalue $\lambda_{-}$and that $\left|\lambda_{-}\right| \leq \max \left[\lambda\left(X^{\Gamma}\right)\right]$ [21]. Suppose now that $X$ fulfills the constraints and has rank larger than 1 . Then $X$ has a separable state $S$ in its support, as each two-dimensional subspace contains at least one separable state [22]. Consider now $y^{2}$ the largest real positive scalar such that $X-y^{2} S \geq 0$. It is easy to verify that the matrix $Y=X-y^{2} S$ also fulfils the four constraints, as $S^{\Gamma}$ is positive due to its separability. Moreover, the value $\operatorname{Tr}\left(S \rho^{\Gamma}\right)=\operatorname{Tr}\left(S^{\Gamma} \rho\right)$ with $S$ separable and $\rho$ entangled is assured to be positive. Therefore the matrix $Y$ will yield a larger value of the cost function. This argument implies that the maximal value of the cost function will be obtained for $X$ rank one. $X$ can therefore be written in the form

$$
X=\left(A \otimes I_{2}\right)|\psi\rangle\langle\psi|\left(A^{\dagger} \otimes I_{2}\right),
$$

and the constraints become $-I_{2} \leq A \leq I_{2}$.

But then the variational characterization of the upper bound (2) becomes exactly equal to the variational characterization of the lower bound (1). This is very surprising as it implies that the proposed 1-LOCC protocol used in deriving the lower bound was actually optimal over all possible LOCC protocols. We have therefore proven the following:

Theorem 2: The optimal trace-preserving LOCC protocol maximizing the fidelity of a given state $\rho$ consists of a 1-LOCC protocol where one party applies a statedependent filter. In case of success, the other party does nothing, and in case of failure, both parties make a pure separable state. The optimal filter and fidelity $F^{*}$ can be found by solving the following convex semidefinite program: maximize

$$
F^{*}=\frac{1}{2}-\operatorname{Tr}\left(X \rho^{\Gamma}\right)
$$

under the constraints

$$
0 \leq X \leq I_{4}, \quad-\frac{I_{4}}{2} \leq X^{\Gamma} \leq \frac{I_{4}}{2} .
$$

$F^{*}>1 / 2$ if $\rho$ is entangled and the optimal $X_{\mathrm{opt}}$ will be of rank 1 , and the filter $A$ can be obtained by making the identification

$$
X_{\mathrm{opt}}=\left(A \otimes I_{2}\right)|\psi\rangle\langle\psi|\left(A^{\dagger} \otimes I_{2}\right)
$$

with $|\psi\rangle=(|00\rangle+|11\rangle) / \sqrt{2}$. This theorem gives us the optimal way of using a mixed state of two qubits for teleportation: the maximal possible fidelity will be obtained when the state is first subjected to the optimal LOCC protocol.

097901-3
The given semidefinite program can be solved exactly if $\rho$ has some symmetry. Indeed, if $\rho^{\Gamma}$ remains invariant under certain symmetry operations, the optimal $X$ can always be chosen such that it has the same symmetry (this follows from a similar argument as the one used during the twirling step in the proof). As an example, we calculate $F^{*}$ for the family of states

$$
\rho(F)=F|\psi\rangle\langle\psi|+(1-F)| 01\rangle\langle 01|
$$

with $F \geq 1 / 3$ the fidelity of the state (these are precisely the states with minimal fidelity for given concurrence [7]). The symmetries under transposition and under the local operations $\sigma_{z} \otimes \sigma_{z}$ and $\operatorname{diag}[1, i] \otimes \operatorname{diag}[1, i]$ imply that $X$ will be real and of the form

$$
X=\left(\begin{array}{cccc}
x_{1} & 0 & 0 & 0 \\
0 & x_{2} & x_{3} & 0 \\
0 & x_{3} & x_{4} & 0 \\
0 & 0 & 0 & x_{5}
\end{array}\right)
$$

Moreover, $x_{1}$ and $x_{5}$ will be equal to zero in the case of an optimal $X$ as otherwise $X$ cannot be rank 1, and a simple optimization problem remains. The optimal filter is readily obtained as $A=\operatorname{diag}[F /(2(1-F)) ; 1]$, and the maximal achievable fidelity $F^{*}$ becomes equal to

$$
\begin{aligned}
& F^{*}(\rho(F))=\frac{1}{2}\left(1+\frac{F^{2}}{4(1-F)}\right) \quad(\text { if } 1 / 3 \leq F \leq 2 / 3), \\
& F^{*}(\rho(F))=F \quad(\text { if } F \geq 2 / 3) .
\end{aligned}
$$

Note that for $F \geq 2 / 3$, no LOCC protocol exists that can increase the fidelity for this class of states. This is true in general: for high fidelities, the fidelity is very close to $(1+N) / 2$ with $N$ the negativity [7] which is an entanglement monotone [23] and can therefore not be increased by LOCC operations.

A quantum state used for teleportation is a special kind of unital or bistochastic quantum channel (see, e.g., $[2,24])$. A unital quantum channel is completely characterized by looking at the image of the Bloch sphere under the action of the channel [25]. In Fig. 1, we depict the images of the Bloch sphere under the action of the teleportation channel obtained by the states $\rho(F)$ of Eq. (3) with $F=0.4$ when the following preprocessing was done: (i) optimal LU preprocessing (implementation of the
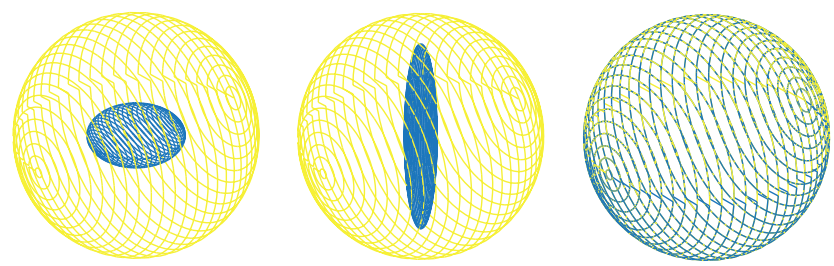

FIG. 1 (color online). The image of the Bloch sphere induced by the teleportation channel with the state $\rho(0.4)$ [Eq. (3)] under optimal LU (left), LOCC (right), and SLOCC (middle) local preprocessing. 
optimal local unitaries such as to maximize singlet fraction [2]); (ii) optimal trace-preserving LOCC transformations (theorem 2); (iii) optimal filtering operations (theorem 1). This gives a nice illustration of the obtained results.

For general states, no analytical method for obtaining an expression of $F^{*}$ is known, and a (simple) semidefinite program has to be solved. It is, however, easy to obtain an explicit lower bound on the optimal $F^{*}$ in terms of the negativity and the concurrence of the original state. This lower bound is obtained by choosing $X$ to be a constant times the subspace spanned by the negative eigenvector $v_{-}$of $\rho^{\Gamma}$. This constant has to be chosen such that the largest eigenvalue of $X^{\Gamma}$ does not exceed $1 / 2$, and it can be shown that this implies that this constant is equal to $1 /\left(1+\sqrt{1-C_{v_{-}}^{2}}\right)$ with $C_{v_{-}}$the concurrence of $v_{-} v_{-}^{\dagger}$. Using the variational characterization of the concurrence of a mixed state $[15,26]$, it is furthermore easy to prove that $C_{v_{-}} \geq N(\rho) / C(\rho)$. Putting all the pieces together, we arrive at the following lower bound for the maximum achievable fidelity $F^{*}$ for an arbitrary state $\rho$ :

$$
\begin{aligned}
\frac{1}{2}\left\{1+\left[N(\rho) / 1+\sqrt{1-\left(\frac{N(\rho)}{C(\rho)}\right)^{2}}\right]\right\} & \leq F^{*}(\rho) \\
& \leq \frac{1}{2}(1+N(\rho)) .
\end{aligned}
$$

The upper bound follows from the fact that the fidelity is bounded above by $(1+N) / 2$ [7] which is an entanglement monotone and can therefore not be increased by LOCC operations.

Before concluding, we show that the maximum achievable fidelity $F^{*}$ belongs to the class of entanglement measures measuring the robustness of entanglement $[14,27]$. To that purpose, we use the fact that to each formulation of a semidefinite program, there exists a dual formulation [20] that yields exactly the same value for the extremum. The dual of (2) can be shown to reduce to: minimize

$$
G=\frac{1}{2}+\frac{1}{2} \operatorname{Tr}(Z)
$$

subject to the constraints

$$
Z \geq 0, \quad(\rho+Z)^{\Gamma} \geq 0 .
$$

Defining the state $\rho_{Z}=Z / \operatorname{Tr}(Z)$, this problem is equivalent to: minimize

$$
G=\frac{1}{2(1-p)}
$$

over all $0 \leq p<1$ and over all states $\rho_{Z}$, subject to the constraint that the state $\rho^{\prime}$,

$$
\rho^{\prime}=(1-p) \rho+p \rho_{Z},
$$

is separable. The minimum value obtained is the maximum achievable fidelity $F^{*}$. As $1 /(1-p)$ is monotonously increasing over $0 \leq p<1$, this problem amounts to finding the state $\rho_{Z}$ such that the weight in the mixture of this state with the original state $\rho$ is minimal, under the constraint that this mixture is separable. The maximal achievable fidelity $F^{*}(\rho)$ is therefore a measure of the minimal amount of mixing required of $\rho$ with another state such that a separable state is obtained.

In summary, we have shown that the fidelity or maximal singlet fraction is not an entanglement monotone but can be made one by defining a new fidelity $F^{*}$ as the maximal achievable one by trace-preserving LOCC operations. These optimal operations were completely determined, and this maximal achievable fidelity $F^{*}$ quantifies the minimal amount of mixing required for a quantum state to destroy its entanglement. The optimal achievable teleportation fidelity is given by $f^{*}=\left(2 F^{*}+1\right) / 3$.

[1] C. H. Bennett et al., Phys. Rev. Lett. 70, 1895 (1993).

[2] M. Horodecki, P. Horodecki, and R. Horodecki, Phys. Rev. A 60, 1888 (1999).

[3] C. H. Bennett, D. P. DiVincenzo, J. A. Smolin, and W. K. Wootters, Phys. Rev. A 54, 3824 (1996).

[4] J. Dehaene, M. Van der Nest, F. Verstraete, and B. De Moor, quant-ph/0207154.

[5] D. Deutsch et al., Phys. Rev. Lett. 77, 2818 (1996).

[6] C. H. Bennett et al., Phys. Rev. Lett. 76, 722 (1996).

[7] F. Verstraete and H. Verschelde, Phys. Rev. A 66, 022307 (2002).

[8] M. Horodecki, P. Horodecki, and R. Horodecki, Phys. Rev. Lett. 78, 574 (1997).

[9] P. Badziag, M. Horodecki, P. Horodecki, and R. Horodecki, Phys. Rev. A 62, 012311 (2000).

[10] E. M. Rains, IEEE Trans. Inf. Theory 47, 2921 (2001).

[11] A. Peres, Phys. Rev. Lett. 77, 1413 (1996).

[12] M. Horodecki, P. Horodecki, and R. Horodecki, Phys. Lett. A 223, 1-8 (1996).

[13] N. Gisin, Phys. Lett. A 210, 151 (1996).

[14] G. Vidal and R. Tarrach, Phys. Rev. A 59, 141 (1999).

[15] F. Verstraete, J. Dehaene, and B. De Moor, Phys. Rev. A 64, 010101(R) (2001).

[16] W.K. Wootters, Phys. Rev. Lett. 80, 2245 (1998).

[17] F. Verstraete and H. Verschelde, quant-ph/0202124.

[18] A. Jamiolkowski, Rep. Math. Phys. 3, 275-278 (1972).

[19] I. Cirac, W. Dür, B. Kraus, and M. Lewenstein, Phys. Rev. Lett. 86, 544 (2001).

[20] L. Vandenberghe and S. Boyd, SIAM Rev. 38, 49 (1996).

[21] F. Verstraete, K. Audenaert, J. Dehaene, and B. De Moor, J. Phys. A 34, 10327 (2001).

[22] M. Lewenstein and A. Sanpera, Phys. Rev. Lett. 80, 2261 (1998).

[23] G. Vidal, J. Mod. Opt., 47, 355-376 (2000).

[24] G. Bowen and S. Bose, Phys. Rev. Lett. 87, 267901 (2001).

[25] C. King and M.-B. Ruskai, IEEE Trans. Inf. Theory 47, 192-209 (2001).

[26] F. Verstraete, J. Dehaene, and B. De Moor, Phys. Rev. A 65, 032308 (2002).

[27] G. Vidal and R. Werner, Phys. Rev. A 65, 032314 (2002). 